\title{
EVIDENCIAÇÃO DE INFORMAÇÕES ESTRATÉGICAS E A VOLATILIDADE DAS AÇÕES
}

\author{
DISCLOSURE OF STRATEGIC INFORMATION AND SHARES VOLATILITY
}

\author{
Nevison Amorim Pereira ${ }^{2}$ \\ Doutorando em Ciências Contábeis pela Universidade Federal de Uberlândia \\ nevison@ufu.br \\ https://orcid.org/0000-0001-7168-0267
}

\section{Marcelo Tavares}

Doutor em Genética e Melhoramento de Plantas pela Universidade de São Paulo Professor do Programa de Pós-Graduação em Ciências Contábeis da Universidade Federal de Uberlândia mtavares@ufu.br https://orcid.org/0000-0003-3008-3460

\section{RESUMO}

Objetivo: O objetivo deste trabalho é analisar a relação entre as informações estratégicas presentes em demonstrações financeiras padronizadas (DFPs) e a volatilidade de ações de empresas brasileiras.

Fundamento: O disclosure (divulgação de informações) é um dos objetivos da Contabilidade e isto representa transparência na relação entre empresas e usuários interessados. Além disto, autores como Murcia e Machado (2013), Malacrida e Yamamoto (2006) e Medeiros e Quinteiro (2005) mostraram que há vantagens econômicas para as empresas evidenciarem suas informações. Como as informações estratégicas estão relacionadas com a competitividade e o mercado de atuação da empresa, esse tipo de informação tem potencial de influenciar o preço das ações. Contudo, a Teoria do Disclosure considera que os gestores divulgam informações de maneira voluntária, quando se há mais benefícios que custos.

Método: A amostra selecionada refere-se a trinta empresas que, no período de 2013 a 2015, negociaram ações na B3 (Brasil, Bolsa, Balcão). Foi efetuada a análise de conteúdo das demonstrações financeiras padronizadas para identificar a evidenciação de informações estratégicas e, em seguida, a volatilidade das ações foram calculadas. Por fim, calculou-se a matriz de correlação e a regressão múltipla entre a variável volatilidade e as demais variáveis: evidenciação estratégica, rentabilidade, endividamento, governança corporativa e tamanho das empresas.

Resultados: Os resultados indicam que, em média, 61,53\% das empresas disponibilizaram informações estratégicas. Para um nível de significância de 5\%, a hipótese de que o disclosure das informações estratégicas impacta negativamente a volatilidade das ações foi aceita. Assim, empresas que eviden-

\footnotetext{
${ }^{1}$ Artigo recebido em: 24/07/2017 . Revisado por pares em: 11/10/2017. Reformulado em: 13/12/2017. Recomendado para publicação em: 13/12/2017 por Luiz Felipe de Araújo Pontes Girão (Editor Geral). Publicado em: 14/02/2018. Organização responsável pelo periódico: UFPB.

${ }^{2}$ Endereço: Av. João Naves de Ávila, 2121 - Santa Mônica, Uberlândia - MG, 38400-902.

DOI: http://dx.doi.org/10.18405/recfin20180207

Os autores agradecem ao editor e aos dois avaliadores anônimos pelos comentários úteis e sugestões perspicazes/relevantes.
} 
ciaram informações estratégicas apresentaram menor volatilidade das ações. Ressalta-se que a variável governança corporativa diverge da literatura, já que não se apresentou significante, e, portanto, não apresenta influência sobre a volatilidade.

Contribuições: Revelar a importância da divulgação das informações estratégicas servindo de incentivos para as empresas aumentarem seu nível de evidenciação.

Palavras-chave: Evidenciação. Informações Estratégicas. Volatilidade.

\section{ABSTRACT}

Objective: The objective of this work is to analyze the relationship between the strategic information present in the standardized financial statements (DFPs) and the volatility of Brazilian companies' shares.

Background: The disclosure (disclosure of information) is one of the objectives of accounting and this represents transparency in the relationship between companies and interested users. In addition, authors such as Murcia and Machado (2013), Malacrida and Yamamoto (2006) and Medeiros and Quinteiro (2005) have shown that there are economic advantages for companies to evidence their information. Since strategic information is related to the company's competitiveness and market, this type of information has the potential to influence stock prices. However, Disclosure Theory considers that managers disclose information on a voluntary basis only when there are more benefits than costs.

Method: The sample selected refers to thirty companies that in the period from 2013 to 2015 traded shares in B3 (Brazil, Bolsa, Balcão). The content analysis of the standardized financial statements was performed to identify the disclosure of the strategic information and then the volatility of the shares was calculated. Finally, statistical methods were used for correlation matrix and multiple regressions, between the variable volatility and the other variables: strategic disclosure, profitability, indebtedness, corporate governance and size.

Results: The results indicate that, on average, $61.53 \%$ of companies provided strategic information. For a significance level of $5 \%$, the hypothesis that the disclosure of strategic information negatively impacts corporate stock volatility was accepted. Thus, companies that showed strategic information showed lower stock volatility. It should be emphasized that the variable corporate governance differs from the literature, since it did not present significant, and, therefore, does not present influence on the volatility.

Contributions: Reveal the importance of disclosure of strategic information serving as incentives for companies to increase their level of disclosure.

Keywords: Disclosure. Strategic Information. Volatility.

\section{INTRODUÇÃO}

O investidor utiliza-se de várias fontes de informações para fazer previsões e tomar suas decisões. Além disso, a informação é relevante para o mercado de capitais e a sua utilidade referese à capacidade de revisar as crenças dos investidores sobre o valor de determinada empresa.

A informação estratégica envolve metas e objetivos das empresas, estratégias do negócio, modelo de governança, informações sobre o ambiente competitivo e principais produtos e mercados de atuação (Epstein \& Birchard, 1999). Está ligado ao desempenho da empresa quanto a sua competitividade e planejamento de mercado, o que pode alterar as crenças dos investidores.

Desta maneira, a divulgação de alguma informação estratégica tem o potencial de alterar as expectativas dos investidores e consequentemente influenciar o preço das ações, pois, conforme a Hipótese do Mercado Eficiente, as variações nos preços incorporam todas as informações e expecta- 
tivas sobre as empresas. Neste cenário, é importante considerar a relação das informações estratégicas e a volatilidade das ações a partir da evidenciação dessas informações nos relatórios contábeis, visto que a Contabilidade é uma das fontes de informação dos investidores.

A informação contábil tem sido um importante instrumento para a redução da assimetria informacional entre as empresas emissoras de ações e os investidores (Ball, 2001). Tais informações contribuem para mitigar as incertezas sobre os investimentos, sendo um dos reflexos esperados, o aumento da liquidez das ações por meio da divulgação de informações corporativas (Murcia \& Machado, 2013).

Estas informações também representam uma ferramenta para a tomada de decisão por várias partes interessadas. Conforme apontam Bakar e Saleh (2011), sua ampla divulgação torna-se essencial, seja no âmbito interno ou externo das organizações.

Para Lopes e Martins (2005), a informação contábil possui o papel de atender à demanda de informações que os investidores necessitam para elaborar estratégias de investimento. Ainda, os preços dos títulos negociados no mercado financeiro refletem as expectativas dos agentes econômicos quanto ao futuro das empresas; e a relação entre as informações contábeis e a variação no preço das ações, mostra que as expectativas do mercado se alteram constantemente.

Ball e Brown (1968) abordaram a relação entre informações contábeis e os retornos das ações avaliando a variação de preços de mercado após a evidenciação de lucros anormais (lucros acima e abaixo das expectativas). Os resultados indicaram taxas de retorno próximas de $7,5 \%$ na data do anúncio dos relatórios contábeis.

Segundo Alencar (2005), como os investidores de ações não têm certeza da credibilidade dos emissores de ações, tendem a diminuir o preço da oferta, elevando o custo de captação de capital próprio por parte das empresas. Espera-se que reduzindo a insegurança dos investidores, por meio de informações, as organizações consigam obter redução do custo de capital. Assim, o disclosure desempenha um papel fundamental, visto que transmite confiança aos investidores.

É importante identificar quais informações devem ser divulgadas, pois, a evidenciação voluntária excede ao requerido por lei e, em muitos casos, acarreta custos extras. A evidenciação de informações não deve limitar-se apenas às financeiras, mas também às estratégicas.

Sanchez, Dominguez e Alvarez (2011) sustentam que a divulgação voluntária de informações estratégicas está gradualmente se tornando uma prática empresarial. Nesse conjunto, incluem-se informações referentes a desenvolvimento de novos produtos, consumidores, empregados, implantação de uma nova fábrica de produção e pesquisa e desenvolvimento (Gruning, 2011). Reforçando os exemplos, Dallabona, Machado e Hein (2013) citam: evidenciação sobre objetivos, benefícios futuros, planejamento estratégico, concorrência, entre outros.

Dallabona et al. (2013) argumentam que as empresas necessitam evidenciar informações de cunho financeiro ou não, objetivando apoiar o investidor em sua tomada de decisão. Murcia e Machado (2013) consideram que a assimetria de informações entre investidores, influencia o preço dos títulos, o número de transações no mercado e, por consequência, a liquidez.

Quando se aumenta e melhora a disponibilidade de informações sobre uma empresa, a assimetria informacional é reduzida, diminuindo também o risco sobre o retorno de um investimento, assim como a volatilidade (Murcia \& Machado, 2013, Garay, González, Guzmán, \& Trujillo, 2013).

A volatilidade é uma variável que reflete o grau de variação de preços de um dado ativo em determinado período de tempo, sendo que, quanto maior a volatilidade, maior a variação do preço e consequentemente maior o risco do papel (Vicente, Araújo, Castro, \& Tavares, 2012). Sendo assim, a volatilidade não é uma variável diretamente observável, sendo influenciada por diversos fatores, que entre eles, configura-se a divulgação de novas informações. 
Os resultados de Tavares, Barbedo e Araújo (2013) evidenciam a influência da assimetria de informação nos retornos diários das carteiras e quanto maior a assimetria de informação destas carteiras, maior a volatilidade de seus retornos.

Apesar dos benefícios do disclosure, Dantas, Zendersky, Santos e Niyama (2005), destacam que as organizações relutam em aumentar o grau de evidenciação sob o argumento de proteção às informações de natureza estratégica. Neste contexto, propõe-se a seguinte questão de pesquisa: a evidenciação de informações estratégicas está associada à volatilidade de ações de empresas brasileiras?

Para responder esta questão, o objetivo do trabalho consiste em analisar a relação entre as informações estratégicas evidenciadas nas demonstrações financeiras padronizadas (DFPs) e a volatilidade das ações de empresas brasileiras. Para tanto, foi necessário identificar a evidenciação dessas informações e mensurar os níveis de endividamento, rentabilidade, tamanho, governança corporativa e a volatilidade das ações das empresas selecionadas.

Pretende-se que a presente pesquisa contribua para um melhor entendimento da importância de se evidenciar informações, visto que elas têm potencial para influenciar o preço das ações, e, assim, auxiliar os investidores a tomarem suas decisões. Do ponto de vista prático, espera-se um aumento do número de disclosure deste tipo de informação por parte das empresas, de forma a serem mais transparentes quanto a seu modelo de negócios.

A realização da pesquisa justifica-se por investigar se a divulgação dessas informações tem aumentado e qual sua relação com a volatilidade. Outra justificativa é a questão da assimetria informacional, pois conforme Murcia e Santos (2009), a divulgação de informações possui um papel importante para o bom funcionamento do mercado de capitais, além de contribuir com a diminuição da assimetria informacional entre investidores e gestores.

Para Malacrida e Yamamoto (2006), o argumento utilizado para que haja maior nível de evidenciação é a esperada repercussão positiva dessas informações, entre as quais: maior retorno e menor custo de captação, visto que o disclosure está relacionado à ideia de transparência corporativa. Ademais, Amihud e Mendelson (1991) destacam que um maior nível de disclosure pode influenciar a liquidez das ações das empresas, o que consequentemente influencia o valor da empresa (Balakrishnan, Billings, Kelly \& Ljungqvist, 2014).

\section{REFERENCIAL TEÓRICO}

\subsection{Informação e Divulgação}

Para Iudícibus, Martins e Gelbcke (2007), o principal objetivo da Contabilidade é prover informações úteis aos seus usuários, e este é atingido por meio do disclosure das demonstrações contábeis. Lopes e Martins (2005) ressaltam que as informações contábeis devem estar intimamente relacionadas com a realidade econômica das empresas, pois só assim, tais informações poderão ser úteis aos agentes financeiros.

Lang e Lundholm (1996) revelam que o aumento da evidenciação das informações está relacionado com o interesse crescente dos analistas e das expectativas de mercado. Evidenciação pode ser obrigatória e não obrigatória; as evidenciações obrigatórias são reguladas por leis e órgãos reguladores específicos. As não obrigatórias, de acordo com Ponte e Oliveira (2004), são mais uma questão de ética e responsabilidade corporativa.

A pesquisa de Armitage e Marston (2008) confirmou que a divulgação de uma empresa é influenciada por uma série de fatores ambientais organizacionais internos e externos, tais como o tamanho da empresa, do mercado, tecnologia, cultura, cotação em bolsa e governança corporativa. Murcia e Santos (2009) identificaram a regulação setorial, o tamanho e as melhores práticas de governança corporativa, como fatores determinantes da divulgação voluntária das companhias abertas no Brasil. 
Os relatórios financeiros publicados pelas empresas podem apresentar diversas informações. No estudo de Murcia e Machado (2013) há a divisão destas informações em duas dimensões: i) econômica: composta por itens relacionados a ambiente de negócios, atividade operacional das empresas, aspectos estratégicos, governança corporativa, informações e índices financeiros; ii) socioambiental: composta por informações financeiras sociais, produtos e serviços, colaboradores, políticas ambientais, impactos dos produtos e processos no meio ambiente, energia e créditos de carbono.

Por meio do relatório anual, páginas web, reuniões de investidores, teleconferências, reuniões privadas, etc., as empresas publicamente fornecem informações a um amplo espectro de partes interessadas, incluindo os atores do mercado de capitais, como: investidores privados, fundos gestores, analistas, instituições públicas, funcionários e clientes. Essencialmente, divulgações voluntárias por meio daquelas mídias, fornecem informações sobre a estratégia da empresa e, portanto, representam os imperativos estratégicos da empresa (Puxty \& Laughlin, 1983).

Lev (2001) considera que as companhias que divulgam seus ativos intangíveis, considerados estratégicos, são mais percebidas pelo mercado e consequentemente, mais valorizadas, porque os investidores fazem suas projeções de maneira mais justa.

A divulgação de informações suplementares das empresas e suas atividades de comunicação, destinadas a criar transparência, são temas muitas vezes abordados a partir de uma perspectiva de accountability. Há evidências apontando que a importância da informação contábil financeira na explicação dos preços das ações tenha sido em geral, decrescente nas últimas décadas, enquanto, outros fatores como estratégia e a propriedade intelectual ganharam relevância nessa explicação (Bukh \& Nielsen, 2011).

Assim, Bukh e Nielsen (2011) rotulam as informações voluntárias normalmente divulgadas por meio de modelos de relatórios de negócios como informação estratégica. Porém, as informações estratégicas envolvem as metas e objetivos da empresa, estratégia do negócio, modelo de governança, principais produtos e serviços, informações sobre o ambiente competitivo e principais mercados de atuação (Epstein \& Birchard, 1999).

Em um estudo sobre a importância da informação não contábil, Eccles e Mavrinac (1995) ressaltaram que a percepção da importância da informação estratégica difere conforme os usuários. A informação transmitida ao mercado é avaliada e interpretada com base na experiência dos atores, colocada em contexto e, finalmente, absorvida no preço das ações por intermédio da produção e divulgação de análises e recomendações, negociação de investidores e divulgações adicionais feitas por esses participantes (Holland \& Johanson, 2003).

Os gestores atuam como portadores de informação corporativa no sentido de que eles podem decidir se desejam ou não divulgar certo tipo de informação. A administração é a mais informada sobre a empresa e divulgará informações relevantes, a menos que isso possa prejudicar as perspectivas futuras da empresa (Bukh \& Nielsen, 2011).

Quando há uma publicação adequada por parte da empresa tende-se aumentar a liquidez das ações no mercado. Assim, têm-se a limitação dos custos de transações, o crescimento da demanda pelas ações e a consequente redução do custo de capital. Por outro lado, quando a divulgação é escassa, aumentam-se as incertezas por parte dos investidores em relação aos ativos dessa empresa (Botosan, 1997).

A ligação entre a informação contábil e o custo de capital das empresas é uma das questões fundamentais em Contabilidade (Lambert, Leuz \& Verrecchia, 2006). Murcia e Santos (2009) apontam estudos em que empresas com maior nível de disclosure possuem menor custo de capital próprio e de terceiros, aumento da liquidez das ações e aumento do acompanhamento por parte dos analistas.

Gruning (2011) investigou a relação entre a divulgação de relatório anual, a liquidez de mercado e o custo do capital para as empresas registradas na Deutsche Börse. Os resultados mostraram 
que a divulgação de relatório anual aumentou a liquidez de mercado e mudou as expectativas dos investidores.

Murcia e Machado (2013) afirmam que a assimetria informacional gera altos custos, baixa liquidez e que o disclosure reduz a assimetria visto que existirão menos investidores com informações privilegiadas e a redução da incerteza acerca do valor da empresa.

Apesar das vantagens apresentadas, Hendriksen e Van Breda (1992) listam algumas razões que levam as empresas a resistirem em apresentar informações relevantes. Entre elas: evitar oferecer vantagens aos concorrentes, dificuldade de compreensão pelos investidores, compensação da relação custo $x$ benefício e ignorância da necessidade dos usuários.

Heinle e Hofmann (2006) examinaram as consequências econômicas ao divulgarem diferentes tipos de informação ao mercado de capitais. Concluíram que a exigência maior de divulgação de informações, por organismos de normalização e reguladores, pode resultar em menor produtividade e reduzir o valor da empresa. Além disso, as empresas podem ser obrigadas a compensar as consequências negativas de divulgação obrigatória, reduzindo a quantidade de divulgação voluntária.

A despeito de haver benefícios e resistência para o disclosure corporativo, algumas organizações são mais transparentes que outras. Neste ponto, o trabalho de Verrecchia (2001) apresenta a Teoria do Disclosure Voluntário, segundo a qual, os gestores e/ou empresas possuem incentivos econômicos para divulgar as informações de maneira voluntária, ou seja, o disclosure voluntário só ocorre quando há mais benefícios que custos.

Na perspectiva de racionalidade econômica, os gestores não divulgarão informações voluntárias que possam prejudicar a si mesmos ou à entidade (Verrecchia, 2001) e a organização tende a divulgar apenas informações positivas (Dye, 2001). No caso das informações negativas, elas serão divulgadas quando os gestores perceberem que serão mais penalizados pelos usuários externos, caso não as divulguem. Nesse sentido, Cruz e Lima (2010) destacam que os gestores decidirão o nível de evidenciação com base no custo a ser incorrido para fornecer as informações.

Portanto, há seleção das informações evidenciadas, implicando em dificuldades com o conteúdo da informação das demonstrações financeiras para explicar a variação do retorno das ações, justificado, em parte, pelo crescimento dos intangíveis. As práticas tradicionais de relatórios corporativos são inadequadas porque não mensuram adequadamente todos os ativos intangíveis que geram valor. Dessa maneira, a divulgação de informações adicionais que apresentem utilidade para o investidor pode influenciar o preço das ações.

\subsection{Estudos Correlatos e Hipótese}

Diversos estudos têm avaliado o efeito da divulgação de informações no desempenho das empresas, que pode ser dividido em desempenho operacional, valor de mercado e retorno das ações (Perez, 1995, Love, 2011).

Vega (2006) avaliou o efeito de aquisição em relação às informações públicas e/ou privadas levando em consideração comerciantes informados, incerteza e uma dispersão de crenças. Os resultados mostraram que quanto mais informações (públicas ou privadas) os investidores têm sobre o verdadeiro valor de um ativo, e quanto mais eles concordam com esta informação, menor o desvio do retorno anormal.

Murcia e Machado (2013) avaliaram o impacto da divulgação corporativa na liquidez das ações de companhias abertas no Brasil. Concluíram que tanto o disclosure econômico quanto o socioambiental impactam a liquidez das ações. 
Ferreira e Martins (2017) analisaram as relações entre divulgação de informações divulgadas na internet, o risco de informação e o retorno das empresas na América Latina. Os resultados evidenciaram que a emissão de American Depository Receipts (ADRs), o tamanho e a liquidez afetam as relações entre disclosure, risco e retorno.

Botosan (1997) estudou a relação entre custo de capital e nível de evidenciação. Elaborou uma medida de nível de disclosure baseada na quantidade de disclosures voluntários de 122 indústrias em 1990. Concluiu que esta relação nem sempre é significante, pois nem todas as empresas apresentaram associação entre maior nível de disclosure e menor custo de capital.

Botosan e Plumlee (2002) identificaram associação negativa entre custo de capital e o nível de divulgação anual, mas uma associação positiva com a divulgação mais tempestiva. As autoras ressaltaram que a informação mais tempestiva aumentou o custo de capital, e elas atribuíram a explicação a um provável aumento na volatilidade.

Silva e Pinheiro (2015) não encontraram evidências de que o nível de disclosure tem relação com o custo de capital, após a convergência das normas brasileiras de contabilidade para as normas internacionais.

Medeiros e Quinteiro (2005) estudaram o disclosure corporativo de trinta empresas brasileiras no período de 2002 a 2004 e a volatilidade de retorno das ações, utilizando-se de um modelo econométrico. Os testes estatísticos confirmaram a hipótese de que firmas com maiores níveis de evidenciação apresentam menor volatilidade dos retornos das ações.

Murcia, Souza, Dill e Costa Júnior (2011) verificaram que o nível de evidenciação corporativo impacta a volatilidade da ação da empresa. Contudo, isso ocorre apenas com o disclosure econômico. Já a divulgação socioambiental e o disclosure total não foram significativos. Isso indica que nem todas as informações divulgadas pelas empresas possuem impacto na volatilidade das ações.

Dallabona et al. (2013) analisaram se existe correlação entre a evidenciação de informações estratégicas, setor de atuação e reuniões proferidas pelo conselho de administração com o desempenho econômico-financeiro de empresas listadas na $\mathrm{B}^{3}$. Os autores concluíram que há correlação apenas entre a evidenciação de informações estratégicas e setor de atuação das empresas, com os indicadores econômico-financeiros e valor de mercado (das ações e da empresa).

Na mesma linha de estudo, Malaquias e Lemes (2015) analisaram a relação entre o nível de evidenciação dos relatórios contábeis de empresas brasileiras não financeiras e a volatilidade no retorno de seus títulos. Eles observaram que o nível de evidenciação apresentou relação positiva e significante com o tamanho das empresas, e que empresas com maiores níveis de evidenciação possuem menor volatilidade nos retornos de seus títulos.

Nota-se que existem incentivos para as empresas aumentarem a divulgação voluntária, assim, formulou-se a seguinte hipótese de pesquisa:

Hipótese da Pesquisa: O disclosure de informações estratégicas impacta negativamente a volatilidade de ações das empresas.

A utilização de informações estratégicas como variável a ser testada, justifica-se porque são consideradas informações não financeiras e que não estão diretamente relacionadas aos instrumentos financeiros, sendo evidenciadas de forma voluntária pelas empresas. Tais informações objetivam benefícios futuros advindos de suas operações (Sanchez et al., 2011).

Acrescenta-se que os países latino-americanos tendem a ter uma baixa proteção legal aos acionistas criando oportunidade para que as empresas se diferenciem adotando práticas de governança corporativa e divulgação (Garay \& Gonzalez, 2008).

Pelos estudos apresentados nesta seção, nota-se que as informações divulgadas se destinam a reduzir a assimetria entre as empresas e o mercado. Isso traz impactos ao custo de capital das empresas, à volatilidade e à liquidez das ações. 


\section{PROCEDIMENTOS METODOLÓGICOS}

As informações estratégicas podem ser divulgadas em vários locais (sites, redes sociais, relatório de sustentabilidade, etc.), mas no caso, as demonstrações financeiras foram utilizadas, pois, a princípio, estas deveriam ser consultadas pelos investidores para avaliarem a possibilidade de investir. Desta forma, foram analisados itens disponíveis nas Demonstrações Financeiras Padronizadas (DFPs), tais como: Notas Explicativas, Relatórios da Administração, Proposta de Orçamento de Capital.

A análise de conteúdo foi utilizada, visto que proporciona confiabilidade e inferência para as observações em contextos específicos (Beretta \& Bozzolan, 2007). As informações foram classificadas por categorias e subcategorias. A Tabela 1 apresenta as subcategorias que foram utilizadas para identificar, nas demonstrações contábeis, os aspectos relacionados à evidenciação estratégica conforme Dallabona et al. (2013). Apesar de passíveis críticas quanto à classificação da subcategoria ser considerada informações estratégicas ou item da estratégia empresarial, elas foram utilizadas como informações estratégicas, pois não foi identificado outro estudo que faça essa diferenciação e as identifique claramente. Assim, preferiu-se uma classificação prévia ao invés de "criar" esses itens.

Tabela 1: Evidenciação estratégica

\begin{tabular}{c|l}
\hline Categoria & \multicolumn{1}{c}{ Subcategoria } \\
\hline \multirow{4}{*}{ Aspectos estratégicos } & Objetivos, missão e metas futuras das empresas. \\
\cline { 2 - 2 } & Planejamento estratégico. \\
\cline { 2 - 2 } & Principais mercados de atuação. \\
\cline { 2 - 2 } & Perspectiva de novos mercados que a empresa pretende atuar. \\
\cline { 2 - 2 } & Descrição do contexto de concorrência. \\
\cline { 2 - 2 } & Pesquisa e desenvolvimento. \\
\cline { 2 - 2 } & Perspectiva de novos investimentos. \\
\cline { 2 - 2 } & Preço dos produtos e serviços das empresas. \\
\hline
\end{tabular}

Para medir a evidenciação estratégica das empresas, as informações foram avaliadas de forma binária: as empresas que divulgaram informações estratégicas receberam nota 1 e as empresas que não divulgaram receberam nota 0 . O nível de evidenciação foi mensurado pelo número de subcategorias divulgadas divididas pelo total de subcategorias (no caso, 8 classes).

A amostra selecionada foi de trinta companhias de capital aberto não financeiras a partir da classificação dos setores da Economatica ${ }^{\circledR}$ que negociam suas ações na $B^{3}$. A escolha das empresas dos setores foi realizada a partir da classe da ação, ou seja, preferencial. Iniciou-se a seleção pelo setor de siderurgia e metalúrgica, visto a sua importância para a economia, além de fornecer produtos base para setores como construção civil, automotivo, bens de capital, máquinas e equipamentos. Como algumas empresas desse setor não apresentaram dados das DFPs disponíveis no sítio eletrônico da $\mathrm{B}^{3}$ (link empresas listadas) foi utilizado complementarmente empresas do setor têxtil e químico.

Segundo Darós, Borba e Abreu (2005), empresas que operam nas bolsas possuem maior transparência e nível de evidenciação. A justificativa para a quantidade de empresa é decorrente da necessidade de se avaliar, manualmente, o disclosure das empresas, optando por analisar um menor número de empresas em um período de tempo maior. Os dados analisados compreenderam os exercícios de 2013 a 2015, pois representam um período (três anos) semelhante ao utilizado no trabalho 
de Murcia e Machado (2013). Desta forma, a coleta abrangeu noventa DFPs (uma DFP para cada empresa em três anos).

Quanto à forma de mensuração da volatilidade, foi adotado procedimento semelhante ao de Malacrida e Yamamoto (2006) e Malaquias e Lemes (2015), conforme equação 1:

$$
\text { Volatilidade }=D P(R) \text {, sendo } R=\ln \left(\frac{P x}{P x-1}\right)
$$

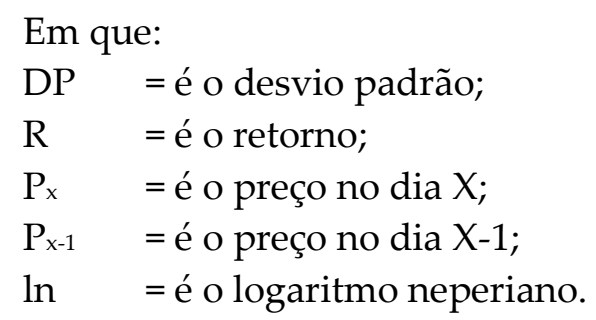

A volatilidade no preço das ações foi medida por períodos de 15, 30 e 45 dias após a data de divulgação das demonstrações contábeis das empresas. A data utilizada como base para início do cálculo da volatilidade foi a data em que as empresas disponibilizaram seus relatórios nos órgãos competentes. Essas datas foram coletadas do site da $\mathrm{B}^{3}$ e as cotações das ações na base de dados Economatica®.

Para realizar o cálculo da volatilidade dos retornos das ações foram utilizadas as cotações das ações preferenciais. A utilização das ações preferenciais baseia-se em Sarlo, Teixeira, Loss e Lopes (2005) que ao analisarem o impacto da divulgação das demonstrações contábeis no retorno de ações preferenciais e ordinárias, demonstraram que para as ações preferenciais, os resultados contábeis representam uma importante fonte de informações ao investidor e que os preços reagem de forma diferente: enquanto os retornos anormais negativos antecipam os resultados a serem divulgados, os retornos anormais positivos variam praticamente no momento da divulgação do resultado.

A função logarítmica foi utilizada para diminuir os efeitos das diferenças de escala na variável "tamanho da empresa" de forma a normalizar a distribuição dos dados. Método semelhante foi utilizado no trabalho de Malaquias e Lemes (2015). A adoção de práticas de governança corporativa visa melhorar a qualidade da informação prestada pelas empresas, reduzindo a assimetria da informação, e, assim, essa variável foi considerada no modelo.

Para análise dos dados foram utilizadas a estatística descritiva, a correlação das variáveis e a regressão linear múltipla. A volatilidade das ações foi considerada a variável dependente. A variável independente foi a evidenciação estratégica. As demais variáveis: rentabilidade, endividamento, governança corporativa e tamanho da empresa foram variáveis de controle, pois, Bushee e Noe (2000), Alencar (2005) e Mota e Pinto (2017) indicaram a utilização de variáveis de controle, pois existem diversos fatores que podem impactar a volatilidade das ações.

Apesar de outras variáveis econômicas do mercado afetar a volatilidade das ações, empregaram-se algumas variáveis utilizadas na literatura, o que permite fazer observações considerando ceteris paribus (todo o mais é constante). Nesse caso, o modelo utilizado é apresentado na equação 2:

$$
\text { Volat }_{i t}=\beta_{0}+\beta_{1} E V I D_{i t}+\beta_{2} R E N T_{i t}+\beta_{3} E N D V_{i t}+\beta_{4} G C_{i t}+\beta_{5} L N A T_{i t}+\varepsilon_{i t}
$$

Em que:

Volat $=$ volatilidade da empresa $i$ no período $t$ calculada pelo desvio-padrão do retorno das ações conforme apresentado anteriormente; 
Evidenciação Estratégica (EVID) $=$ as informações estratégicas da empresa $i$ no tempo $t$ foi mensurada de forma binária, sendo que as empresas que divulgaram receberam nota 1 e as empresas que não divulgaram nota 0 . Teoricamente é esperada uma relação negativa entre a evidenciação estratégica e a volatilidade, mas ainda não há evidências empíricas desta relação;

Rentabilidade $($ RENT) = a proxy utilizada foi ROE (Retorno sobre Patrimônio Líquido) representada pela divisão do lucro líquido pelo valor do patrimônio líquido da empresa $i$ no tempo $t$. É esperada uma relação negativa entre rentabilidade e volatilidade, conforme Murcia et al. (2011);

Endividamento $(E N D V)=$ representado pela divisão do passivo total pelo ativo total da empresa $i$ no tempo $t$. É esperada uma relação positiva entre endividamento e volatilidade conforme Murcia et al. (2011);

Governança Corporativa (GC) = o nível de governança corporativa foi classificado de forma binária, sendo que as empresas que fazem parte de um grupo diferenciado de governança conforme classificação da B3 (Nível 1, Nível 2 ou Novo Mercado) receberam nota 1, e as empresas que não fazem parte receberam nota 0 . É esperada uma relação negativa entre volatilidade e nível de governança (Paiva, Oliveira, \& Peixoto, 2015);

Tamanho da empresa (LNAT) = a proxy utilizada é o logaritmo do ativo da empresa $i$ no tempo $t$. É esperada uma relação negativa entre tamanho da empresa e volatilidade, conforme Paiva et al. (2015);

$\varepsilon=$ é o termo de erro referente à empresa $i$ no tempo $t$, tendo por premissa distribuição normal com média zero e variância constante e finita;

$\beta=$ são os coeficientes das variáveis explicativas.

Para identificação do modelo de regressão mais adequado para o estudo foram realizados os testes de Chow (pooling versus efeito fixo) e Breusch-Pagan (pooling versus efeito aleatório), com resultados de Prob $(\mathrm{F})=$ 0,07 e Prob (chi2) = 0,41 respectivamente, sendo o mais indicado o efeito pooling.

Como os dados apresentavam distribuição normal, para identificar problemas de autocorrelação e multicolinearidade realizou-se, respectivamente, o teste de correlação de Pearson e o teste VIF (Variance Inflation Factors). A maior correlação encontrada foi entre as variáveis endividamento e volatilidade $(0,442)$, pressupondo a não existência de autocorrelação, pois, segundo Wooldridge (2006), caso o coeficiente de correlação ultrapasse 0,60 pode vir a desestabilizar a estimação. $O$ teste VIF para as variáveis ficou entre 1,437 e 2,141, ou seja, sem multicolinearidade porque todos os valores ficaram abaixo de 10 (Gujarati, 2006).

O teste de Durbin-Watson (DW) verifica a ausência de autocorrelação serial dos resíduos. Segundo Guajarati (2006), o atendimento do pressuposto ocorre quando os valores da estatística DW se posicionam próximos a dois. O resultado foi 2,051. Por fim, a homocedasticidade foi verificada pelo teste Pesarán-Pesarán. Para atendimento do pressuposto (dados homogêneos em sua distribuição), o valor da significância deverá ser superior a 0,05 no referido teste ANOVA. A significância do teste indicou 0,162 .

\section{RESULTADOS E DISCUSSÃO}

As médias da volatilidade dos retornos das ações se apresentaram muito próximas nos diferentes períodos analisados ( 15 dias $=0,0218 ; 30$ dias $=0,0219 ; 45$ dias $=0,0218)$. Os valores médios indicaram baixa volatilidade, dado que o valor máximo é 0,1074 (Tabela 2).

Em relação à variável independente, em média, 61,53\% das empresas disponibilizaram evidenciação estratégica (Tabela 2). Resultado coerente com Dantas et al. (2005), que afirmam que as empresas ainda relutam em divulgar as informações estratégicas. 
Tabela 2: Estatística descritiva

\begin{tabular}{lllll}
\hline & Min. & Máx. & Média & Desvio Padrão \\
\hline D15 & 0,0039 & 0,1074 & 0,0218 & 0,0152 \\
D30 & 0,0082 & 0,1061 & 0,0219 & 0,0142 \\
D45 & 0,0090 & 0,1000 & 0,0218 & 0,0130 \\
EVID & 0,0000 & 1,0000 & 0,6153 & 0,2652 \\
RENT & $-6,2845$ & 6,4538 & 2,2093 & 2,0458 \\
ENDV & 0,3386 & 1,0500 & 0,6458 & 0,1698 \\
GC & 0,0000 & 1,0000 & 0,7231 & 0,1820 \\
LNAT & 12,9572 & 20,4051 & 17,1871 & 1,5241 \\
\hline
\end{tabular}

D15, D30 e D45=volatilidade, respectivamente 15, 30 e 45 dias posteriores à data de divulgação das DFPs; LNAT = logaritmo do ativo da empresa; RENT = rentabilidade medida pelo ROE; ENDV = divisão passivo total pelo ativo total; EVID = evidenciação das informações estratégicas; GC = nível de governança corporativa.

Todavia, nota-se uma evolução do nível de evidenciação estratégica das empresas analisadas conforme o passar dos anos (Tabela 3), fato que pode ser explicado pela crescente exigência do mercado em receber cada vez mais informações (Lang \& Lundholm, 1996).

Tabela 3: Nível de Evidenciação

\begin{tabular}{cc}
\hline Ano & Evidenciação (média) \\
\hline 2013 & 0,5625 \\
2014 & 0,6125 \\
2015 & 0,6708 \\
\hline
\end{tabular}

Resultado que corrobora Sanchez et al. (2011) ao dizerem que a divulgação das informações estratégicas estão se tornando mais frequentes. Para Ball (2001), o acréscimo de informações torna os relatórios contábeis mais relevantes, gerando benefícios aos investidores e demais usuários.

Por meio da matriz de correlação (Tabela 4) verificaram-se relações positivas e negativas entre a variável dependente e as variáveis independentes, porém observa-se baixa correlação entre elas.

A associação entre volatilidade das ações e a evidenciação estratégica apresentou índice negativo e significante nos três períodos (Tabela 4), mesmo resultado encontrado por Malaquias e Lemes (2015), Medeiros e Quinteiro (2005), Malacrida e Yamamoto (2006) e Murcia e Machado (2013).

Conforme esperado, a volatilidade dos retornos das ações apresentou relação inversa e estatisticamente significante com o tamanho (Paiva et al., 2015). Também teve relação negativa com a rentabilidade das empresas, assim como uma relação positiva com o nível de endividamento, em conformidade a Murcia et al. (2011).

Ainda que a governança corporativa tenha apresentado relação positiva com o nível de evidenciação estratégica, conforme Murcia e Santos (2009), e uma associação negativa com a volatilidade em D30 e D45, mesma relação encontrada no trabalho de Paiva et al. (2015), essa variável não foi significativa com nenhuma das variáveis (Tabela 4). 
Tabela 4: Matriz de correlação de Pearson das variáveis

\begin{tabular}{lllllllll}
\hline \multicolumn{1}{l}{ D15 } & D30 & D45 & LNAT & RENT & ENDV & EVID & GC \\
\hline LNAT & $-0,261^{*}$ & $-0,331^{*}$ & $-0,311^{*}$ & 1,000 & & & & \\
\hline RENT & $-0,420^{*}$ & $-0,527^{*}$ & $-0,539^{*}$ & $0,296^{*}$ & 1,000 & & & \\
\hline ENDV & $0,426^{*}$ & $0,442^{*}$ & $0,429^{*}$ & $-0,089$ & $-0,215^{*}$ & 1,000 & & \\
\hline EVID & $-0,370^{*}$ & $-0,425^{*}$ & $-0,395^{*}$ & 0,013 & $0,311^{*}$ & $-0,333^{*}$ & 1,000 & \\
\hline GC & 0,009 & $-0,037$ & $-0,018$ & 0,147 & $-0,051$ & 0,186 & 0,010 & 1,000 \\
\hline
\end{tabular}

D15, D30 e D45=volatilidade, respectivamente 15, 30 e 45 dias posteriores à data de divulgação das DFPs; LNAT = logaritmo do ativo da empresa; RENT = rentabilidade medida pelo ROE; ENDV = divisão passivo total pelo ativo total; EVID = evidenciação das informações estratégicas; GC = nível de governança corporativa.

* correlação significativa ao nível de 5\%.

O nível de explicação do modelo, representado pelo $\mathrm{R}^{2}$, foi 30,4\% para volatilidade de 15 dias, $43,7 \%$ para a volatilidade de 30 dias, e $41,4 \%$ para a volatilidade de 45 dias (Tabela 5). Estes valores indicam quanto da volatilidade das ações é explicado pelo modelo atual.

Os resultados dos coeficientes de determinação apresentaram-se maiores em relação ao trabalho de Murcia e Machado (2013). Isso pode ser explicado parcialmente pela medição do nível de evidenciação estratégica, que foi mensurado através de um número reduzido de itens (apenas oito) e pelo tamanho da amostra.

As variáveis de controle tamanho (LNAT), rentabilidade (RENT) e governança corporativa (GC) apresentaram relação negativa com a volatilidade, enquanto a variável endividamento foi positiva (Tabela 5).

A governança corporativa não foi significativa em nenhum dos períodos, resultado que não corrobora com parte dos trabalhos da literatura. Malacrida e Yamamoto (2006) identificaram a governança corporativa como um meio de controle e acompanhamento dos negócios, não somente pelos acionistas como também pelos seus gestores, proporcionando sanar possíveis conflitos de agência, gerando, de forma significativa, impacto na volatilidade das ações. Paiva et al. (2015) encontraram relação negativa e significante, pois pertencer aos níveis diferenciados de governança corporativa reduz a volatilidade das ações da firma.

Tabela 5: Resultados da estimação do modelo

\begin{tabular}{|c|c|c|c|c|c|c|c|c|}
\hline \multicolumn{3}{|c|}{ Variável Dependente: D15 } & \multicolumn{3}{|c|}{ Variável Dependente: D30 } & \multicolumn{3}{|c|}{ Variável Dependente: D45 } \\
\hline & coef. & p-valor & & coef. & p-valor & & coef. & p-valor \\
\hline (Constante) & 0,0417 & 0,0229 & (Constante) & 0,0500 & 0,0014 & (Constante) & 0,0431 & 0,0030 \\
\hline EVID & $-0,0108$ & $0,0479 *$ & EVID & $-0,0116$ & $0,0157^{*}$ & EVID & $-0,0088$ & $0,0490^{*}$ \\
\hline RENT & $-0,0019$ & $0,0117^{*}$ & RENT & $-0,0024$ & $0,0002^{*}$ & RENT & $-0,0024$ & $0,0001^{*}$ \\
\hline ENDV & 0,0271 & $0,0025^{*}$ & ENDV & 0,0245 & $0,0011^{*}$ & ENDV & 0,0219 & $0,0018^{*}$ \\
\hline GC & $-0,0011$ & 0,6960 & GC & $-0,0022$ & 0,3423 & GC & $-0,0017$ & 0,4416 \\
\hline LNAT & $-0,0015$ & 0,1144 & LNAT & $-0,0018$ & $0,0295^{*}$ & LNAT & $-0,0014$ & 0,0659 \\
\hline $\mathrm{R}^{2}$ ajustado & & 0,3048 & $\mathrm{R}^{2}$ ajustado & & 0,4374 & $\mathrm{R}^{2}$ ajustado & & 0,4149 \\
\hline Prob. (F) & & 0,0000 & Prob. (F) & & 0,0000 & Prob. (F) & & 0,0000 \\
\hline
\end{tabular}

D15, D30 e D45= volatilidade, respectivamente 15, 30 e 45 dias posteriores à data de divulgação das DFPs; LNAT = logaritmo do ativo da empresa; RENT = rentabilidade medida pelo ROE; ENDV = divisão passivo total pelo ativo total; EVID = evidenciação das informações estratégicas; GC = nível de governança corporativa.

* Significante ao nível de $5 \%$. 
Os resultados (Tabela 5) estão de acordo com Mota e Pinto (2017) que não identificaram significância ao avaliarem se empresas com níveis diferenciados de governança corporativa possuem melhores níveis de disclosure voluntário, através da divulgação nas contas oficiais do Twitter e com Ferreira (2012), que não encontrou evidências de que empresas participantes dos níveis de governança são mais eficientes que as do mercado tradicional, quando a média dos dois grupos foram comparadas.

Analisando o coeficiente da variável tamanho da empresa, este se encontra de acordo com Paiva et al. (2015) e Malaquias e Lemes (2015) quanto a empresas maiores apresentarem menor volatilidade no preço das ações. Porém, a variável só foi significativa com trintas dias (Tabela 5).

A princípio, o investidor elegerá as ações com maior liquidez e rentabilidade, sendo esperado que ações mais rentáveis sejam mais voláteis devido ao risco, pois segundo Halov e Heider (2005) o investidor externo conhece menos informação sobre o risco de seu investimento, se o valor do ativo tiver grande flutuação. Com base em Paiva et al. (2015) a variável rentabilidade apresentou-se conforme esperado (relação negativa com a volatilidade).

Ainda de acordo com o esperado, o endividamento (ENDV) apresentou significância e relação positiva com a volatilidade das ações (Tabela 5). Assim, quanto maior o endividamento maior a volatilidade. O que, em parte, pode ser explicado por Andrade, Salazar, Calegário e Silva (2009), em que empresas com maior participação de capital de terceiros são vistas como de maior risco. No estudo de Teixeira (2011), o endividamento não se mostrou significativo com a volatilidade, ou seja, não impactou a volatilidade das ações.

A relação do nível de evidenciação estratégica e a volatilidade, objetivo dessa pesquisa, é negativa e significativa. Portanto, quanto maior for o nível de evidenciação menor será a volatilidade. Essa relação encontrada está alinhada os trabalhos de Medeiros e Quinteiro (2005), Malacrida e Yamamoto (2006), Dallabona et al. (2013), Garay et al. (2013), Malaquias e Lemes (2015), Verrecchia (2001) e Botosan e Plumlee (2002). Desta forma, a hipótese de que a evidenciação de mais informações aos investidores diminui suas incertezas, reduzindo a volatilidade dos retornos das ações foi aceita.

\section{CONSIDERAÇÕES FINAIS}

No mercado de capitais, o risco de um investimento em ações é representado por sua volatilidade. A priori, uma das formas de reduzir a volatilidade seria divulgar mais informações a respeito do futuro da empresa (Murcia et al., 2011).

Nesse sentido, este estudo teve como objetivo analisar a relação entre as informações estratégicas presentes nas DFPs e a volatilidade das ações de empresas brasileiras. Tendo essa finalidade foram selecionadas para a análise de conteúdo as DFPs de trinta empresas e calculadas as volatilidades das ações.

Inicialmente, constatou-se que, em média, 61,53\% das empresas consultadas disponibilizaram evidenciação estratégica em suas demonstrações. Posteriormente, utilizou-se da análise de correlação e do modelo de regressão múltipla para testar a hipótese. Após a aplicação do modelo de regressão com efeito pooling, conclui-se pela aceitação da hipótese. Ou seja, empresas que apresentam evidenciação das informações estratégicas apresentam menor volatilidade das ações, revelando assim, a esperada importância dada pelo mercado às demonstrações voluntárias apresentadas pelas empresas. Os resultados confirmam estudos nacionais (Medeiros \& Quintero, 2005, Malacrida \&Yamamoto, 2006, Murcia et al., 2011; Malaquias \& Lemes, 2015) e internacionais (Lev, 2001, Garay et al., 2013).

Apesar de ser significativa a relação entre essas variáveis, ressalta-se que apenas em torno de 1\% volatilidade das ações é explicada pela variável evidenciação estratégica, de modo que outras 
variáveis econômicas do mercado devem ser consideradas pelos analistas e/ou gestores das empresas.

Apesar da divulgação das informações estratégicas representarem um risco para as empresas, visto que tais evidenciações podem ser utilizadas pelos seus concorrentes, espera-se que os resultados sirvam para diminuir a resistência à evidenciação, pois as empresas devem apresentar suas informações de maneira a serem mais transparentes e diminuir o custo de capital próprio. Garay et al. (2013) enfatizam que maiores níveis de disclosure estão positivamente relacionados com melhores desempenhos operacionais, que tendem a ser melhor avaliados no mercado. Ademais, KudlawiczFranco, Bach e Silva (2016) destacam que a assimetria da informação influencia no desempenho das empresas.

Dado o tamanho da amostra e a quantidade de subcategorias utilizadas para mensurar a evidenciação estratégica, os resultados e conclusões obtidos estão limitados ao conjunto de empresas analisadas, apesar dos resultados estarem em conformidade com a literatura consultada.

O trabalho contribui para a literatura existente sobre disclosure voluntário no sentido que confirma o resultado esperado entre divulgação de informações estratégicas e a volatilidade das ações, mas difere de estudos anteriores quanto a variável governança corporativa que não foi significativa, ou seja, não afeta a volatilidade.

Como sugestão para pesquisas futuras, recomenda-se estender o período de análise e a amostra, visto que nem todas as variáveis foram significativas e podem estar associadas apenas a amostra utilizada neste estudo. Com isso, existe a possibilidade de que, aumentando-se o espaço de tempo e a quantidade de variáveis, as conclusões aqui apresentadas possam sofrer alterações. Outra sugestão é incluir novas variáveis e definir a variável evidenciação estratégica com base em outros elementos que reflitam as estratégias da empresa, identificando por meio de análise fatorial, quais são as mais relevantes. Uma vez que foram analisadas apenas as DFPs, caberia analisar outros meios de divulgação de informação, como os websites das empresas.

\section{REFERÊNCIAS}

Alencar, R. C. (2005). Custo do capital próprio e nível de disclosure nas empresas brasileiras. Brazilian Business Review, 2(1), 1-12. Recuperado em 15 de novembro de 2016 de: http://www.spell.org.br/documentos/ver/20538/custo-do-capital-proprio-e-nivel-de-disclosure.

Amihud, Y., \& Mendelson, H. (1991). Liquidity, Asset Prices and Financial Policy. Financial Analysts Journal, 47, 56-66. Retrieved December 15, 2016, from http://people.stern.nyu.edu/lpederse/courses/LAP/papers/TransactionCosts/AmihudMendelsonFAJ91.pdf.

Andrade, L. P. D., Salazar, G. T., Calegário, C. L. L., \& Silva, S. S. (2009). Corporate governance: an analysis of board of directors in relation with the value of market and performance of the Brazilian companies. Revista de Administração Mackenzie, 10(4), 4-31. Recuperado em 2 de dezembro de 2016 de: http://www.scielo.br/scielo.php?pid=S1678-69712009000400002\&script=sci_abstract.

Armitage, S., \& Marston, C. (2008). Corporate disclosure, cost of capital and reputation: evidence from finance directors. The British Accounting Review, 40, 314-336. Retrieved November 30, 2016, from http://www.sciencedirect.com/science/article/pii/S0890838908000516.

Bakar, N. B. A., \& Saleh, Z. (2011). Incentives for Disclosure of Accounting Information in Public Sector: A Literature Survey. International Research Journal of Finance and Economics, 75(1), 24-38. 
Retrieved December 15, 2016, from http://www.academia.edu/1039957/Incentives_for_Disclosure_of_Accounting_Information_in_Public_Sector_A_Literature_Survey.

Balakrishnan, K., Billings, M. B., Kelly, B., \& Ljungqvist, A. (2014). Shaping Liquidity: On the Causal Effects of Voluntary Disclosure. Journal of Finance, 69(5), 2237-2278. Retrieved July, 18, 2017, from http://www.nber.org/papers/w18984.

Ball, R. (2001). Infrastructure Requirements for an Economically Efficient System of Public Financial Reporting and Disclosure. Papers on Financial Services, Brooking Institution Press, 127-169. Retrieved December 17, 2016, from https://scholar.google.com/citations?user=TEVgnyQAAAAJ\&hl=en.

Ball, R., \& Brown, P. (1968). An empirical evaluation of accounting income numbers. Journal of Accounting Research, 6, 159-178. Retrieved January 5, 2017, from http://econ.au.dk/fileadmin/Economics_Business/Education/Summer_University_2012/6308_Advanced_Financial_Accounting/Advanced_Financial_Accounting/0/Ball_Brown_JAR_1968.pdf.

Beretta, S., \& Bozzolan, S. (2007). Quality versus quantity: The case of forward-looking disclosure. Journal of Accounting, Auditing and Finance, 23(3), 333-376. Retrieved November 18, 2016, from http://journals.sagepub.com/doi/pdf/10.1177/0148558X0802300304.

Botosan, C. A. (1997). Disclosure Level and the cost of equity capital. The Accounting Review, 72(3), 323-349. Retrieved January 5, 2017, from https://www.jstor.org/stable/248475.

Botosan, C. A., \& Plumlee, A. (2002). A re-examination of disclosure level and the cost of equity capital. Journal of Accounting Research, 40(1), 21-40. Retrieved July 20, 2017, from https://papers.ssrn.com/sol3/papers.cfm?abstract_id=279309.

Bukh, P. N., \& Nielsen, C. (2011). The supply and demand for strategic information: A case study of a large medical device company. Journal of Human Resource Costing \& Accounting, 15(1), 50-77. doi: https://doi.org/10.1108/14013381111125314.

Bushee, B. J., \& Noe, C. F. (2000). Corporate disclosure practices, institutional investors, and stock return volatility. Journal of Accounting Research, 38, 203-207. Retrieved January 10, 2017, from https://www.jstor.org/stable/2672914?seq=1\#page_scan_tab_contents.

Cruz, C., A., Lima, G. (2010). Reputação corporativa e nível de disclosure das empresas de capital aberto no Brasil. Revista Universo Contábil, 6(1), 85-101. Recuperado em 28 junho de 2017 de: http://proxy.furb.br/ojs/index.php/universocontabil/article/view/838/1151. doi:10.4270/ruc.2010105.

Dallabona, L. F., Machado, M. M., \& Hein, N. (2013). A influência da divulgação de informações estratégicas, setor de atuação e reunião do conselho de administração com o desempenho econômico de empresas listadas na BM\&FBOVESPA. Anais do SIMPOI, São Paulo/SP. 
Dantas, J. A., Zendersky, H. C., Santos, S. C., \& Niyama, J. K. (2005). A dualidade entre os benefícios do disclosure e a relutância das organizações em aumentar o grau de evidenciação. Revista Economia e Gestão, 5(11), 56-76. Recuperado em 2 de janeiro de 2017 de: http://www.spell.org.br/documentos/ver/36406/.

Darós, L. L., Borba, J. A., \& Abreu, A. F. de. (2005). Evidenciação de instrumentos financeiros derivativos nas demonstrações contábeis: uma comparação entre as informações divulgadas no Brasil e nos Estados Unidos pelas empresas brasileiras emitentes de American Depositary Receipts - ADR's. Anais do EnANPAD, Brasília/DF.

Dye, R. (2001). An evaluation of 'essays on disclosure' and the disclosure literature in accounting. Journal of Accounting and Economics, 32, 181-235.

Eccles, R. G. \& Mavrinac, S. (1995). Improving the corporate disclosure process. Sloan Management Review, 36(4), 11-25.

Epstein, M. J. \& Birchard, B. (1999). Counting what counts: Turning corporate accountability to competitive advantage. Reading, Massachusetts: Perseus Books.

Ferreira, R. do N. (2012). Governança Corporativa e Desempenho: Uma Análise em Empresas Brasileiras de Capital Aberto. Tese doutorado, Universidade Federal de Lavras, Lavras, MG, Brasil.

Ferreira, T. S. V., \& Martins, O. S. (2017). Relationship analysis between disclosure on the internet, risk and return in Latin American companies. Revista de Administração Mackenzie, 18(2), 154-183. Retrieved July 15, from http://www.scielo.br/pdf/ram/v18n2/1678-6971-ram-18-02-0154.pdf. http://dx.doi.org/10.1590/1678-69712016/administracao.v18n2p154-183.

Garay, U., \& González, M. (2008). Corporate governance and firm value: the case of Venezuela. Corporate Governance an International Review Journal, 16(3), 194-209. Retrieved July 11, from http://onlinelibrary.wiley.com/doi/10.1111/j.1467-8683.2008.00680.x/abstract

Garay, U., González, M., Guzmán, A., \& Trujillo, M. A. (2013). Internet-based corporate disclosure and market value: Evidence from Latin America. Emerging Market Review, 17(1), 160-168. Retrieved July 11, from https://papers.ssrn.com/sol3/papers.cfm?abstract_id=2146937.

Gruning, M. (2011). Capital markets implications of corporate disclosure: German evidence. BuR Bussiness Research, 4(1), 48-72. Retrieved January 20, from http://link.springer.com/article/10.1007/BF03342726.

Gujarati, D. N. (2006). Econometria básica. 4. ed. Rio de Janeiro: Elsevier.

Halov, N., \& Heider, F. (2005). Capital Structure, Risk and Asymmetric information. Social Science Research Network. Retrieved January 20, 2017 from http://citeseerx.ist.psu.edu/viewdoc/download?doi=10.1.1.197.7304\&rep=rep1\&type=pdf.

Heinle, M. S.; \& Hofmann, C. (2006). Soft information and the stewardship value of accounting disclosure. Social Science Research Network. Retrieved September 10, 2016 from http://papers.ssrn.com/sol3/papers.cfm?abstract_id=1334327. 
Hendriksen, E. S., \& Van Breda, M. F. (1992). Accounting Theory. Burr Ridge: Irwin.

Holland, J. B. \& Johanson, U. (2003). Value relevant information on corporate intangibles - creation, use, and barriers in capital markets - between a rock and a hard place. Journal of Intellectual Capital, 4(4), 465-86.

Iudícibus, S. de, Martins, E., \& Gelbcke, E. R. (2007). Manual de contabilidade das sociedades por ações (aplicável às demais sociedades) (7a. ed.). São Paulo: Atlas.

Kudlawicz-Franco, C., Bach, T. M., \& Silva, E. D. da. (2016). Assimetria de informação e desempenho: Um estudo em empresas de capital aberto no Brasil. Revista de Gestão dos Países da Língua Portuguesa, 15(2). Recuperado em 5 de janeiro de 2017 de: http://www.scielo.mec.pt/scielo.php?script=sci_abstract\&pid=S1645-44642016000200003\&lng=pt\&nrm=iso.

Lambert, R., Leuz, C., \& Verrecchia, R. E. (2006). Accounting Information, Disclosure, and the Cost of Capital. Social Science Research Network. Retrieved September 09, 2016 from http://papers.ssrn.com/sol3/papers.cfm?abstract_id=823504.

Lang, M. H., \& Lundholm, R. J. (1996). Corporate Disclosure Policy and Analyst Behaviour. The Accounting Review, 71(4), 467-492. Retrieved January 20, 2017, from https://www.jstor.org/stable/248567?seq=1\#page_scan_tab_contents.

Lev, B. (2001). Measuring the value of Intellectual Capital. Ivey Business Journal. New York, March / April, 16-20.

Lopes, A. B., \& Martins, E. (2005). Teoria da contabilidade: uma nova abordagem. São Paulo: Atlas.

Love, I. (2011). Corporate governance and performance around the world: what we know and what we don't. The World Bank Research Observer, 26(1), 42-70.

Malacrida, M., \& Yamamoto, M. (2006). Governança corporativa: Nível de evidenciação das informações e sua relação com a volatilidade das ações do Ibovespa. Revista Contabilidade e Finanças, $17,65-79$.

Malaquias, R. F., \& Lemes, S. (2015). Evidenciação e Volatilidade: Testes com equações estruturais. Base - Revista de Administração e Contabilidade da Unisinos, 12(2), 96-109. Recuperado em 30 de novembro de 2016 de: http://www.spell.org.br/documentos/ver/36766/.

Medeiros, O., \& Quinteiro, L. (2005). Disclosure of accounting information and stock return volatility in Brazil. Social Science Research Network. Recuperado em 18 novembro de 2016 de: https://papers.ssrn.com/sol3/papers.cfm?abstract_id $=835726$.

Mota, S. L., \& Pinto, S. K. M. (2017). A utilização do twitter na análise do disclosure voluntário das empresas brasileiras com níveis diferenciados de governança corporativa. Revista Evidenciação Contábil \& Finanças. 5(1), 22-38. Recuperado em 15 de janeiro de 2017 de: http://periodicos.ufpb.br/ojs2/index.php/recfin/article/view/30647. doi:10.18405/recfin20170102. 
Murcia, F. D., \& Machado, M. V. (2013). Impacto do nível de disclosure corporativo na liquidez das ações de companhias abertas no Brasil. Revista Contabilidade Vista E Revista, 24(3), 54-77. Recuperado em 30 de novembro de 2016 de: revistas.face.ufmg.br/index.php/contabilidadevistaerevista/article/download/.../pdf_57.

Murcia, F. D., \& Santos, A. (2009). Fatores determinantes do nível de disclosure voluntário das companhias abertas no Brasil. Revista de Educação e Pesquisa em Contabilidade, 3(2), 72-95. Recuperado em 6 de janeiro de 2017 de: http://www.repec.org.br/index.php/repec/article/view/68. doi: http://dx.doi.org/10.17524/repec.v3i2.68.

Murcia, F. D., Souza, F. C., Dill, R. P., \& Costa Junior, N. C. A. (2011). Impacto do nível de disclosure corporativo na volatilidade das ações de companhias abertas no Brasil. Revista de Economia e Administração, 10(2), 196-218.

Paiva, J. F. M., Oliveira, N. A., \& Peixoto, F. M. (2015) A relação entre conselho de administração, desempenho, valor e risco no mercado brasileiro de ações. Revista de educação e Pesquisa em contabilidade, 9(1), 25-44. Recuperado em 15 de janeiro de 2017 de: http://www.repec.org.br/index.php/repec/article/view/1168.

Perez, M. M. (1995). Métricas de retorno e a geração de valor ao acionista. Unisanta - Law and Social Science, 4(2), 189-202.

Ponte, V. M. R., \& Oliveira, M. C. (2004). A prática da evidenciação de informações avançadas e não obrigatórias nas demonstrações contábeis das empresas brasileiras. Revista Contabilidade $\mathcal{E}$ Finanças, 15(36). Recuperado em 15 de dezembro de 2016 de: http://www.scielo.br/scielo.php?script=sci_arttext\&pid=S1519-70772004000300001.

Puxty, T. \& Laughlin, R. (1983). A rational reconstruction of the decision-usefulness criterion. Journal of Business Finance \& Accounting, 10(3), 543-560. Retrieved October 20, 2017, from http://onlinelibrary.wiley.com/doi/10.1111/j.1468-5957.1983.tb00452.x/abstract.

Sanchez, I. M. G., Dominguez, L. R., \& Alvarez, I. G. (2011). Corporate governance and strategic information on the internet. Accounting, Auditing \& Accountability Journal, 24, 471-501. Retrieved January 12, 2017, from http://www.emeraldinsight.com/doi/abs/10.1108/09513571111133063.

Sarlo, A. N., Teixeira, A. J. C., Loss, L., \& Lopes, A. B. (2005). O diferencial no impacto dos resultados contábeis nas ações ordinárias e preferenciais no mercado brasileiro. Revista Contabilidade $\mathcal{E}$ Finanças, 16(37), 46-58. Recuperado em 3 de janeiro de 2017 de: http://www.scielo.br/scielo.php?script=sci_arttext\&pid=S1519-70772005000100004.

Silva, D. A., \& Pinheiro, L. E. T. (2015). O impacto do nível de disclosure sobre o custo de capital próprio das companhias abertas brasileiras. Revista de Contabilidade e Organizações, 25, 73-88. Recuperado em 18 de julho de 2017 de: http://www.revistas.usp.br/rco/article/viewFile/97726/107583.

Tavares, M. L. F., Barbedo, C. H. S., \& Araújo, G. S. (2013). A Influência da Assimetria de Informação no Retorno e na Volatilidade das Carteiras de Ações de Valor e de Crescimento. ? [Working paper nº 312], Banco Central do Brasil, 1-25. 
Teixeira, E. E. M. (2011). Relação entre as expectativas econômicas publicadas no relatório FOCUS e a volatilidade dos retornos das ações mais negociadas do IBOVESPA. Tese de doutorado Universidade Federal de Minas Gerais, Belo Horizonte, MG, Brasil.

Vega, C. (2006). Stock price reaction to public and private information. Journal of Financial Economics, 82, 103-133. Retrieved December 7, 2016, from http://www.sciencedirect.com/science/article/pii/S0304405X06000444.

Verrecchia, R. E. (2001). Essays on disclosure. Journal of Accounting and Economics. 32(1-3), 97-180. Retrieved July 10, 2017, from http://www.sciencedirect.com/science/article/pii/S0165410101000258.

Vicente, J. V. M.; Araújo, G. S.; Castro, P. B. F.; \& Tavares, F. N. (2012). Avaliando a Volatilidade Diária dos Ativos: a hora da negociação importa? [Working paper n⿳o 297], Banco Central do Brasil, 1-30.

Wooldridge, J. M. (2006). Introdução à econometria: uma abordagem moderna (5a. ed.). São Paulo: Pioneira Thompson Learning 